\title{
Case Report \\ Severe Diltiazem Poisoning Treated with Hyperinsulinaemia-Euglycaemia and Lipid Emulsion
}

\author{
Nadine Monteiro, ${ }^{1}$ Joana Silvestre, ${ }^{1,2}$ João Gonçalves-Pereira, ${ }^{1,2}$ \\ Camila Tapadinhas, ${ }^{1}$ Vitor Mendes, ${ }^{1}$ and Pedro Póvoa ${ }^{1,2}$ \\ ${ }^{1}$ Polyvalent Intensive Care Unit, São Francisco Xavier Hospital, West Lisbon Hospital Centre, 1449-005 Lisbon, Portugal \\ ${ }^{2}$ Faculty of Medical Sciences, New University of Lisbon, 1169-056 Lisbon, Portugal \\ Correspondence should be addressed to Nadine Monteiro; nadine_rodrigues@hotmail.com
}

Received 10 April 2013; Accepted 5 May 2013

Academic Editors: C. Diez, M. Egi, C. Lazzeri, K. Lenz, W. S. Park, and K. S. Waxman

Copyright (C) 2013 Nadine Monteiro et al. This is an open access article distributed under the Creative Commons Attribution License, which permits unrestricted use, distribution, and reproduction in any medium, provided the original work is properly cited.

Introduction. Calcium channel blockers (CCBs) drugs are widely used in the treatment of cardiovascular diseases. CCB poisoning is associated with significant cardiovascular toxicity and is potentially fatal. Currently, there is no specific antidote and the treatment of CCB poisoning is supportive; however, this supportive therapy is often insufficient. We present a clinical case of severe diltiazem poisoning and the therapeutic approaches that were used. Case Report. A 55-year-old male was admitted to the intensive care unit (ICU) after voluntary multiple drug intake, including extended release diltiazem $(7200 \mathrm{mg})$. The patient developed symptoms of refractory shock to conventional therapy and required mechanical ventilation, a temporary pacemaker, and renal replacement therapy. Approximately 17 hours after drug intake, hyperinsulinaemia-euglycaemia with lipid emulsion therapy was initiated, followed by progressive haemodynamic recovery within approximately 30 minutes. The toxicological serum analysis $12 \mathrm{~h}$ after drug ingestion revealed a diltiazem serum level of $4778 \mathrm{ng} / \mathrm{mL}$ (therapeutic level: $40-200 \mathrm{ng} / \mathrm{mL}$ ). Conclusions. This case report supports the therapeutic efficacy of hyperinsulinaemia-euglycaemia and lipid emulsion in the treatment of severe diltiazem poisoning.

\section{Introduction}

Diltiazem is a nondihydropyridine L-type calcium channel blocker (CCB) which is widely used in the treatment of cardiovascular diseases. The prescription of CCB has increased significantly in recent years, $[1,2]$ and concomitantly the number of cases of voluntary and involuntary poisoning.

In 2011, the American Association of Poison Control Centers reported 1995 deaths from exposure to toxic substances, 1689 of which were from medications (84.7\%) [3]. Following analgesics and antidepressants, cardiovascular drugs were the most often involved. Of these drugs, CCB were most commonly used [3].

Calcium channel blockers overdose can cause life-threatening effects, such as bradycardia, atrioventricular (AV) block, hypotension, metabolic acidosis, and shock that is often refractory to conventional therapy.

The treatment of CCB poisoning has been limited to organ support measures. The importance of hyperinsulinaemia-euglycaemia and lipid emulsion therapy has recently been recognised in the treatment of these patients [412]. Traditionally, these approaches are used as late salvage therapy in cases of CCB poisoning when other measures have failed.

We hereby present a clinical case of a patient with severe diltiazem poisoning in which hyperinsulinaemia-euglycaemia and lipid emulsion therapy contributed to haemodynamic stabilisation.

\section{Clinical Case}

A 55-year-old male was admitted to the emergency room (ER) with a low level of consciousness approximately two hours after voluntary multiple drug ingestion, including diltiazem $(7200 \mathrm{mg})$, perindopril $(150 \mathrm{mg})$, simvastatin $(280 \mathrm{mg})$, and escitalopram $(600 \mathrm{mg})$.

The patient presented a past history of essential arterial hypertension, ischemic cardiac disease, dyslipidaemia, and major depression. 
TABLE 1: Laboratory data.

\begin{tabular}{|c|c|c|c|c|}
\hline & Hospital admission & UCI admission & 12 hrs after ICU admission & 48 hrs after ICU admission \\
\hline Red blood cells $\left(\times 10^{12} / \mathrm{L}\right)$ & 4.72 & 3.9 & 3.94 & 4.01 \\
\hline Haemoglobin (g/dL) & 14.0 & 11.5 & 11.7 & 12.1 \\
\hline White cell count $\left(\times 10^{9} / \mathrm{L}\right)$ & 10.5 & 23.8 & 19.0 & 19.1 \\
\hline Platelet count $\left(\times 10^{9} / \mathrm{L}\right)$ & 224 & 216 & 127 & 93 \\
\hline Prothrombin time (sec) & 10.9 & 11.8 & 13.7 & 11.0 \\
\hline C-reactive protein $(\mathrm{mg} / \mathrm{dL})$ & 0.41 & 0.49 & 11.3 & 9.8 \\
\hline Urea $(\mathrm{mg} / \mathrm{dL})$ & 40 & 49 & 50 & 23 \\
\hline Creatinine $(\mathrm{mg} / \mathrm{dL})$ & 1.22 & 1.79 & 2.47 & 1.70 \\
\hline Glucose (mg/dL) & 225 & 306 & 275 & 186 \\
\hline Potassium $(\mathrm{mmol} / \mathrm{L})$ & 3.56 & 4.54 & 3.85 & 4.2 \\
\hline Sodium (mmol/L) & 135 & 137 & 137 & 135 \\
\hline Calcium (mg/dL) & 8.5 & 6.6 & 9.7 & 8.8 \\
\hline Alanine transaminase $(\mathrm{U} / \mathrm{L})$ & 36 & 49 & & \\
\hline Aspartate transaminase $(\mathrm{U} / \mathrm{L})$ & & 28 & & \\
\hline$\gamma$-glutamyltransferase $(\mathrm{U} / \mathrm{L})$ & 55 & 119 & & \\
\hline Total bilirubin (mg/dL) & 0.37 & 0.88 & 0.44 & 0.46 \\
\hline
\end{tabular}

TABLE 2: Evolution of arterial blood gases in the ICU.

\begin{tabular}{|c|c|c|c|c|c|c|}
\hline & $0 \mathrm{~h}\left(\mathrm{FiO}_{2}: 0.3\right)$ & $4 \mathrm{~h}^{*}\left(\mathrm{FiO}_{2}: 0.6\right)$ & $9 \mathrm{~h}\left(\mathrm{FiO}_{2}: 0.8\right)$ & $10 \mathrm{~h}\left(\mathrm{FiO}_{2}: 0.6\right)$ & $12 \mathrm{~h}\left(\mathrm{FiO}_{2}: 0.5\right)$ & $48 \mathrm{~h}^{* *}\left(\mathrm{FiO}_{2}: 0.4\right)$ \\
\hline $\mathrm{pH}$ & 7.241 & 7.242 & 7.195 & 7.263 & 7.35 & 7.396 \\
\hline $\mathrm{PaCO}_{2}(\mathrm{mmHg})$ & 46.1 & 35.5 & 29.3 & 31.1 & 31.4 & 38.8 \\
\hline $\mathrm{PaO}_{2}(\mathrm{mmHg})$ & 68.6 & 72.5 & 207 & 188 & 217 & 134 \\
\hline $\mathrm{HCO}^{-3}(\mathrm{mEq} / \mathrm{L})$ & 16.7 & 15.5 & 12.6 & 15.1 & 18.8 & 23.8 \\
\hline Lactate $(\mathrm{mmol} / \mathrm{L})$ & 4.3 & 5.7 & 9.4 & 6.7 & 3.4 & 0.8 \\
\hline
\end{tabular}

* Intubated and invasive mechanical ventilation was initiated.

${ }^{* *}$ Extubated.

On the hospital admission, he was comatose with a Glasgow Coma Score (GCS) of 8, a blood pressure (BP) of $77 / 44 \mathrm{mmHg}$, and a heart rate (HR) of $48 \mathrm{bpm}$.

The patient began treatment with intravenous fluid, atropine, glucagon, sodium bicarbonate, and calcium gluconate. Severe bradycardia and hypotension persisted, for which a dopamine infusion was initiated (maximum dose: $7.5 \mathrm{mcg} / \mathrm{kg} / \mathrm{min})$.

Laboratory evaluation data are presented in Table 1. Arterial blood gases $\left(\mathrm{FiO}_{2}\right.$ 0.3) revealed a metabolic acidosis $\left(\mathrm{pH}\right.$ 7.306, $\mathrm{PCO}_{2} 34.0 \mathrm{mmHg}, \mathrm{PO}_{2} 90.3 \mathrm{mmHg}, \mathrm{HCO}_{3}$ $17.6 \mathrm{mmol} / \mathrm{L}$, and lactate $3.9 \mathrm{mmol} / \mathrm{L}$ ).

An electrocardiogram was performed and showed a sinus rhythm with 48 bpm; a first-degree atrioventricular block; and the pattern of a complete right bundle branch block.

The condition progressed to refractory shock, and the patient developed an acute renal failure with oliguria. At that time he was admitted in the intensive care unit (ICU).

Aggressive fluid resuscitation and vasopressor support with dopamine, norepinephrine, epinephrine, terlipressin, and dobutamine were initiated with poor hemodynamic response. Progressive worsening of lactic acidosis was observed (Table 2). An intravenous (iv) calcium infusion of $2 \mathrm{~g} / \mathrm{h}$ was instituted, and renal replacement therapy was initiated (continuous veno-venous hemodiafiltration at effluent rates of $35 \mathrm{~mL} / \mathrm{Kg} / \mathrm{h}$ ) without significant change in his cardiovascular failure.

The patient's level of consciousness deteriorated even further (GCS: 5), and he was subsequently tracheally intubated, and invasive mechanical ventilation was initiated. The patient evolved with a severe bradycardia, needing a temporary pacemaker.

After approximately 9 hours in the ICU, refractory hypotension persisted. At that time, a 20\% lipid emulsion infusion was prescribed at $0.5 \mathrm{~mL} / \mathrm{Kg} / \mathrm{h}$ rate, plus (iv) short-acting human insulin at increasing doses reaching a maximum dose of $45 \mathrm{U} / \mathrm{h}$ and $30 \%$ dextrose in water infusion adjusting infusion rate for the maintenance of euglycaemia.

Approximately $30 \mathrm{~min}$ after the initiation of these supportive measures, progressive haemodynamic improvement was observed with the normalisation of lactic acidaemia (Table 2), which allowed a gradual weaning of vasopressor support as well as in the recovery of own cardiac rhythm.

After 48 hours in ICU, the patient was successfully weaned from ventilatory and vasopressor support.

Despite diuresis recovery, the need for renal replacement therapy persisted until haemodynamic stabilisation was observed. Continuous veno-venous haemodiafiltration was stopped and deescalated to intermittent hemodialysis. The patient was discharged from ICU on the 4 th day of hospital 
stay. The patient experienced full recovery of renal function and returned home after 3 days.

The plasma concentration of diltiazem $12 \mathrm{~h}$ after ingestion was $4778 \mathrm{ng} / \mathrm{mL}$ (therapeutic level: $40-200 \mathrm{ng} / \mathrm{mL}$ ), that is to say almost 24 times above the upper limit of the therapeutic range.

\section{Discussion}

In this clinical case, the authors describe a severe CCB poisoning (near $24 \times$ the upper volume of the therapeutic range) that results in a severe refractory shock with multiple organ failure that only recovered with the hyperinsulinaemiaeuglycaemia and lipid emulsion treatment.

The cardiovascular effects of CCB poisoning involve the excessive blockage of the L-type calcium channels in the myocardial cell membranes of the cardiac electrical conduction system and the vascular smooth muscle tissue, thereby preventing the entry of calcium into the cells. Therefore, cardiac inotropism, dromotropism, and chronotropism are reduced along with vascular tone. In addition, CCB inhibits the influx of calcium into pancreatic beta cells and peripheral tissue which leads to the decreased excretion of insulin and peripheral resistance to the action of CCB $[7,13,14]$. Conventional therapy for $\mathrm{CCB}$ poisoning includes the administration of fluids, calcium salts, glucagon, and vasopressors $[5,6,15-$ 17].

Extracorporeal elimination by conventional haemofiltration and dialysis is not recommended because these agents bind to plasma proteins and have a large volume of distribution. The molecular adsorbent recirculating system (MARS) was successfully used in one case report of severe diltiazem poisoning [18]. However, this technique is expensive and is not always available in a timely manner.

In our case, there was rapid deterioration of the clinical condition and refractoriness to conventional therapy which was rapidly reversed after the prescription of hyperinsulinaemia-euglycaemia and lipid infusion therapy.

In normal circumstances, myocardial cells use the oxidation of free fatty acids as an energy substrate for aerobic metabolism. In CCB poisoning, the uptake of free fatty acids by the myocardium is decreased, and the myocardium uses glucose as an energy substrate. However, the decrease in tissue perfusion secondary to the excessive blockage of vascular calcium channels complicates the distribution of glucose into the tissue. Simultaneously, hypoinsulinaemia and insulin resistance prevent the uptake of glucose by myocardial cells and vascular smooth muscle, thus limiting the use of glucose as an energy substrate. The lack of energy substrate exacerbates cardiovascular depression which is already compromised by the blocking of the calcium channels.

These mechanisms led to the hypothesis that the administration of high-dose insulin to treat CCB poisoning could compensate for hypoinsulinaemia and insulin resistance and as a result could interrupt the vicious cycle that is responsible for progressive haemodynamic deterioration and, ultimately, patient death. The efficacy and safety of this treatment have been demonstrated in several cases of CCB poisoning [6$8,10,12]$.
Therapeutic hyperinsulinaemia-euglycaemia consists of a continuous infusion of high-dose regular short-acting insulin $(0.5-1 \mathrm{UI} / \mathrm{kg})$ with concomitant glucose infusion that is titrated to maintain glycaemia within normal limits, which may necessitate a glucose dosage of $15-30 \mathrm{~g} / \mathrm{h}$.

Lipid emulsion has been used to treat poisoning by local anaesthetics. There is insufficient data to support the use of lipid emulsion as a first-line option; however, this therapy has been used as salvage therapy in pharmaceutical poisoning by other lipophilic drugs, particularly CCBs $[9,12]$. The exact mechanism of action of this treatment is not known. The most widely accepted theory is that the emulsion acts as a "lipid sink," surrounding a lipophilic drug molecule and rendering it ineffective.

\section{Conclusions}

The combination of hyperinsulinaemia-euglycaemia and lipid emulsion therapy was effective for the haemodynamic recovery of a patient with refractory cardiogenic shock secondary to severe diltiazem poisoning. The early prescription of these therapies in patients with $\mathrm{CCB}$ poisoning may improve their prognosis.

\section{References}

[1] I. Kurnatowska, J. Królikowski, K. Jesionowska et al., "Prevalence of arterial hypertension and the number and classes of antihypertensive drugs prescribed for patients late after kidney transplantation," Annals of Transplantation, vol. 17, no. 1, pp. 5057, 2012.

[2] K. A. J. Al Khaja, R. P. Sequeira, and V. S. Mathur, "Rational pharmacotherapy of hypertension in the elderly: analysis of the choice and dosage of drugs," Journal of Clinical Pharmacy and Therapeutics, vol. 26, no. 1, pp. 33-42, 2001.

[3] A. C. Bronstein, D. A. Spyker, L. R. Cantilena Jr., B. H. Rumack, and R. C. Dart, "2011 Annual report of the American Association of Poison Control Centers' National Poison Data System (NPDS): 29th annual report," Clinical Toxicology, vol. 50, no. 10, pp. 911-1164, 2012.

[4] A. Anusheree, S. W. Yu, Abdul Rehman, and J. Q. Henkle, "Hyperinsulinemia euglycemia therapy for calcium channel blocker overdose," The Texas Heart Institute Journal, vol. 39, no. 4, pp. 575-578, 2012.

[5] N. Abeysinghe, J. Aston, and S. Polouse, "Diltiazem overdose: a role for high-dose insulin," Emergency Medicine Journal, vol. 27, no. 10, pp. 802-803, 2010.

[6] B. Mégarbane, S. Karyo, and F. J. Baud, "The role of insulin and glucose (hyperinsulinaemia/euglycaemia) therapy in acute calcium channel antagonist and $\beta$-blocker poisoning," Toxicological Reviews, vol. 23, no. 4, pp. 215-222, 2004.

[7] P. E. R. Lheureux, S. Zahir, M. Gris, A. S. Derrey, and A. Penaloza, "Bench-to-bedside review: hyperinsulinaemia/euglycaemia therapy in the management of overdose of calciumchannel blockers," Critical Care, vol. 10, no. 3, article 212, 2006.

[8] S. L. Greene, I. Gawarammana, D. M. Wood, A. L. Jones, and P. I. Dargan, "Relative safety of hyperinsulinaemia/euglycaemia therapy in the management of calcium channel blocker overdose: a prospective observational study," Intensive Care Medicine, vol. 33, no. 11, pp. 2019-2024, 2007. 
[9] B. J. Wilson, J. S. Cruikshank, K. L. Wiebe, V. C. Dias, and M. C. Yarema, "Intravenous lipid emulsion therapy for sustained release diltiazem poisoning: a case report," Journal of Population Therapeutics and Clinical Pharmacology, vol. 19, no. 2, pp. e218e222, 2012.

[10] L. Min and K. Deshpande, "Diltiazem overdose haemodynamic response to hyperinsulinaemia-euglycaemia therapy: a case report," Critical Care and Resuscitation, vol. 6, no. 1, pp. 28-30, 2004.

[11] S. J. Stellpflug, S. J. Fritzlar, J. B. Cole, K. M. Engebretsen, and J. S. Holger, "Cardiotoxic overdose treated with intravenous fat emulsion and high-dose insulin in the setting of hypertrophic cardiomyopathy," Journal of Medical Toxicology, vol. 7, no. 2, pp. 151-153, 2011.

[12] V. Montiel, T. Gougnard, and P. Hantson, "Diltiazem poisoning treated with hyperinsulinemic euglycemia therapy and intravenous lipid emulsion," European Journal of Emergency Medicine, vol. 18, no. 2, pp. 121-123, 2011.

[13] J. A. Kline, R. M. Raymond, J. D. Schoroeder, and J. A. Watts, "The diabetogeic effects of acute verapamil poisoning," Toxicology and Applied Pharmacology, vol. 145, no. 2, pp. 357-362, 1997.

[14] D. F. Blackburn and T. W. Wilson, "Antihypertensive medications and blood sugar: theories and implications," Canadian Journal of Cardiology, vol. 22, no. 3, pp. 229-233, 2006.

[15] S. D. Salhanick and M. W. Shannon, "Management of calcium channel antagonist overdose," Drug Safety, vol. 26, no. 2, pp. 6579, 2003.

[16] P. D. Pearigen and N. L. Benowitz, "Poisoning due to calcium antagonists. Experience with verapamil, diltiazem and nifedipine," Drug Safety, vol. 6, no. 6, pp. 408-430, 1991.

[17] C. R. DeWitt and J. C. Waksman, "Pharmacology, pathophysiology and management of calcium channel blocker and $\beta$-blocker toxicity," Toxicological Reviews, vol. 23, no. 4, pp. 223-238, 2004.

[18] M. Belleflamme, P. Hantson, T. Gougnard et al., "Survival despite extremely high plasma diltiazem level in a case of acute poisoning treated by the molecular-adsorbent recirculating system," European Journal of Emergency Medicine, vol. 19, no. 1, pp. 59-61, 2012. 


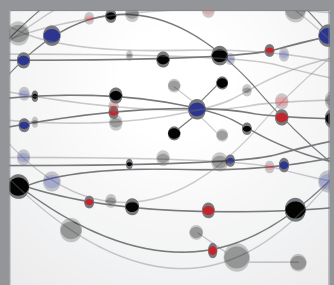

The Scientific World Journal
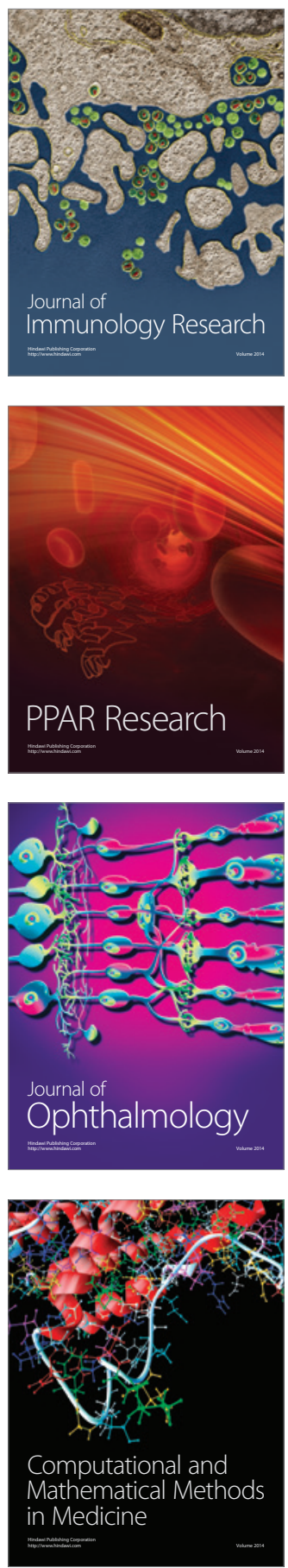

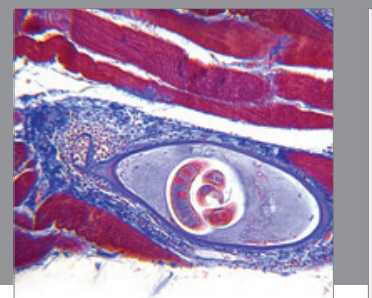

Gastroenterology

Research and Practice
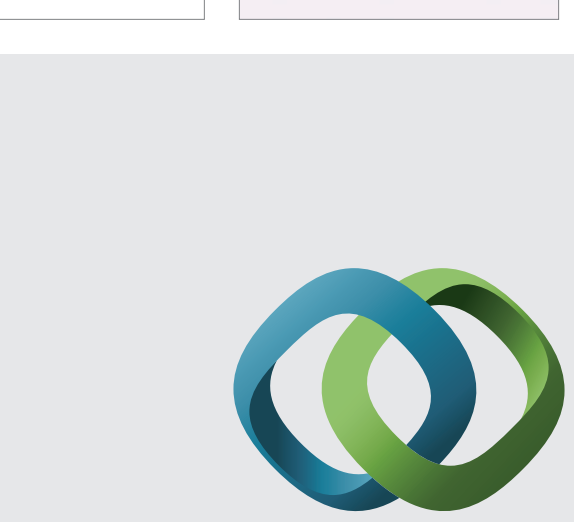

\section{Hindawi}

Submit your manuscripts at

http://www.hindawi.com
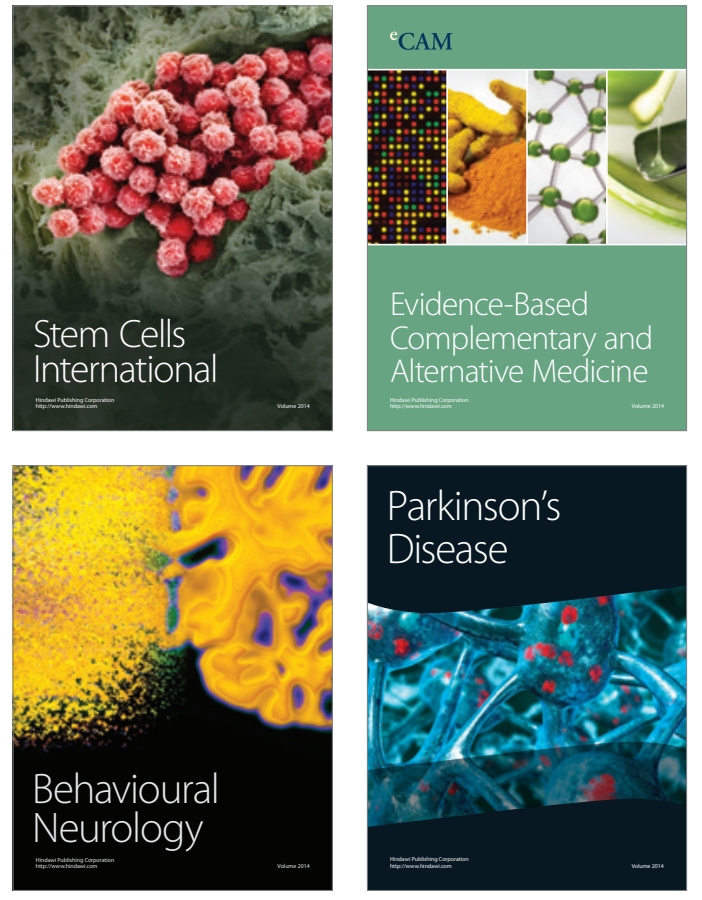
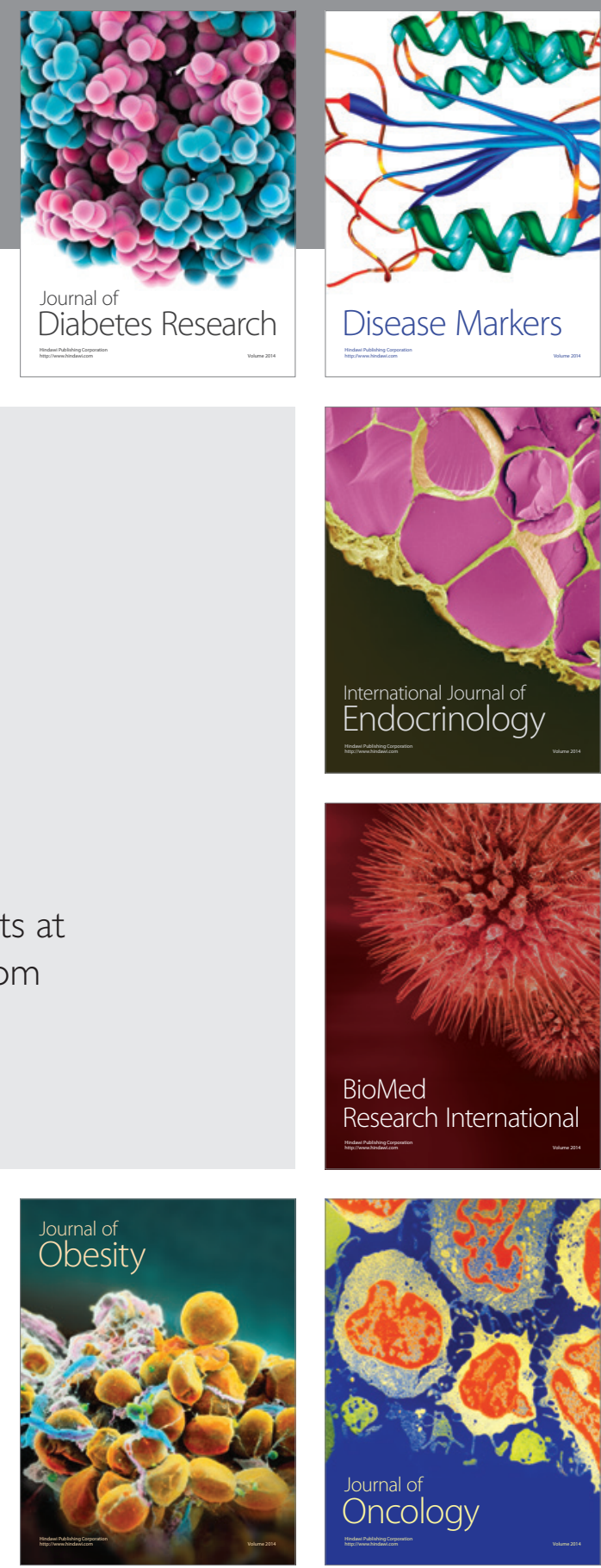

Disease Markers
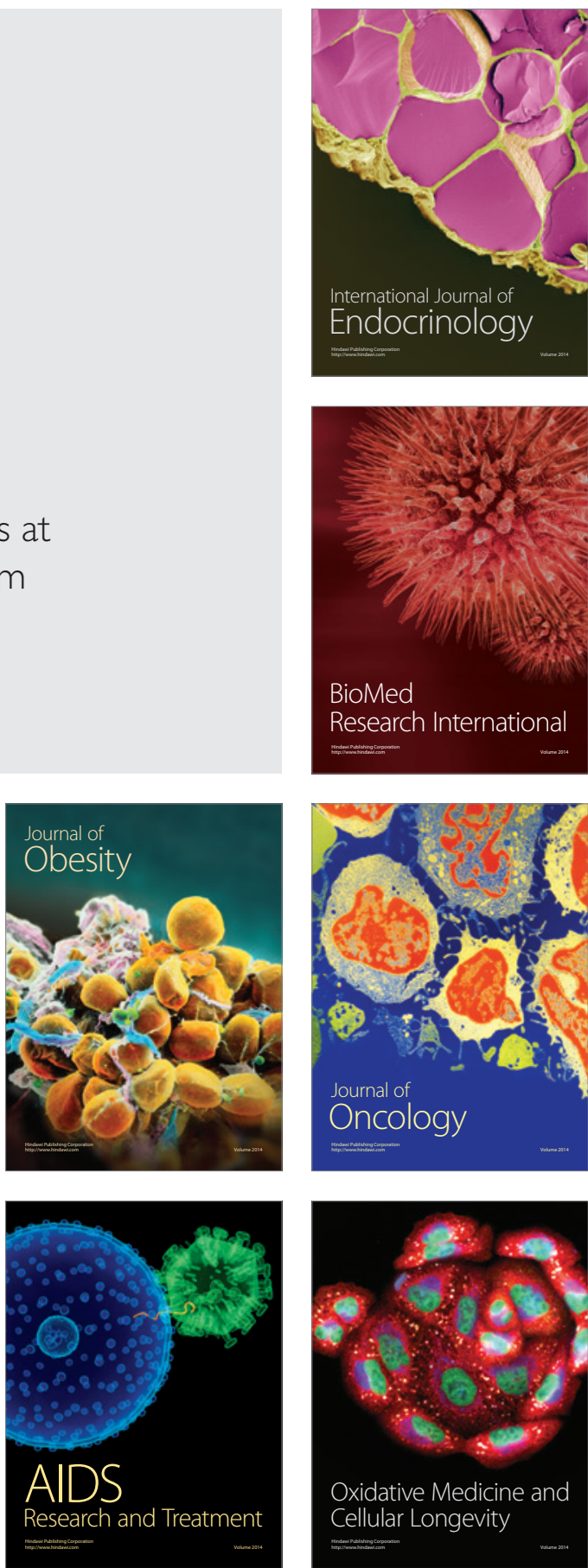